\title{
Strict Lyapunov functions for model reference adaptive control: application to Lagrangian systems
}

\author{
Antonio Loría Elena Panteley Mohamed Maghenem
}

\begin{abstract}
Model reference adaptive control is a wellunderstood and popular method used in the case when the plant's constant parameters are unknown and must be identified. The related literature is very rich and there exist many proofs of stability and convergence. Lyapunov functions for such systems, having the property that the total derivative is negative definite, are, however, very scarce. In this note we use the Mazenc construction to design a simple strict Lyapunov function in a rather intuitive manner, based on a first-choice function whose derivative is negative semidefinite. Furthermore, we provide, for the first time in the literature, a Lyapunov function for a popular passivity-based adaptive controller for Lagrangian systems.
\end{abstract}

\section{INTRODUCTION}

Driven by concrete control problems of physical systems (specifically, mechanical, electrical and electromechanical) analysis and design of adaptive control systems has been, in turn, a steering force in control theory and practice for decades now. An early significant breakthrough was the certaintyequivalence principle, which states that in the case of parametric uncertainty, one can use the same controller that would be used in the case that all the parameters of the system were known, replacing their values with estimates. Then, an adaptation law is designed to update such estimates. In view of its simplicity, model-reference adaptive control [1] is among the most popular control and estimation techniques, but it also poses significant challenges to stability analysis, due to its inherent nonlinear and time-varying nature; even in the case when the plant to be controlled is linear and autonomous [2].

Two distinct problems of convergence analysis naturally appear: of the tracking errors and of the parameter estimation errors. For the former, a commonly-used method is based on properties of signals in $\mathcal{L}_{p}$ spaces and the popular Barbălat's lemma [3]. Establishing convergence of the parameter estimation errors, however, is a much more challenging task of analysis. The one condition that is invoked under various control schemes is known as persistency of excitation and it was introduced in [4] in the context of identification of discretetime linear systems. While conserving its conceptual substance, this condition has taken different mathematical forms in the literature, depending on the context: for continuous-time linear systems necessary and sufficient conditions for uniform convergence are presented in [5], [6], [7]; for non-uniform convergence, in [6]; for nonlinear time-varying systems, in [8] and [9] —see also [10]; for linear parameterized systems in

A. Loría and E. Panteley are with the CNRS. LSS-CentraleSupelec, 3 Rue Joliot Curie, 91192, France. E. Panteley is also with ITMO University, Kronverkskiy av. 49, Saint Petersburg, 197101, Russia. M. Maghenem is with the University of California at Santa Cruz.

This article is supported by Univ Paris Saclay and by the Government of Russian Federation (grant 074-U01).
[11]. Its equivalence to uniform complete observability is discussed in detail in [12] and its equivalence to detectability, in the realm of nonlinear time-varying systems, was established in [13]. In the specific context of adaptive control, [10] and [14] provide a thorough study.

Now, because adaptive control systems are inherently nonlinear, a crucial question beyond that of convergence is whether the origin is uniformly asymptotically, or exponentially, stable. For linear systems the latter may be established upon the scrutiny of the state transition matrix [15], or using the concept of uniform complete observability and output injection techniques [12]; see also [16] where an explicit stability bound is computed. For nonlinear systems, however, exponential stability is impossible [17]; one can "only" achieve uniform global asymptotic stability [18].

Certainly, the most direct method of stability analysis is Lyapunov's second. Yet, constructing a strict Lyapunov function for adaptive control systems has eluded the research community, at least until [19] where, to the best of our knowledge, the first strict Lyapunov function for nonlinear systems reminiscent of model-reference-adaptive control was proposed. Ref. [19] relies on the neat Mazenc construction, which was introduced in [20] and is described in great detail in [21]. Roughly speaking, the starting point is a Lyapunov function whose derivative is negative in part of the state variables only; then, a function of this non-strict Lyapunov function is constructed. See also [22], [23].

In this paper we employ the Mazenc construction on a narrower class of systems than the one considered in [19], [22]. The systems that we study here appear in model-referenceadaptive control of nonlinear systems and other passivitybased control techniques. For the purpose of illustration we also propose, as far as we know for the first time, a strict Lyapunov function for the popular tracking adaptive Slotine \& Li controller for Lagrangian systems originally proposed in [24], and which continues to inspire solutions to more contemporary problems such as mutual synchronization of Lagrangian systems — see e.g. [25], [26], [27], [28].

The rest of this paper is organized as follows. In the next section we describe the class of systems that we consider. In Section II-C we present our main results, the utility of which is illustrated through a concrete example in Section III. Finally, we conclude with some remarks in Section IV.

\section{PROBlem SETting AND ITS SOLUTION}

\section{A. Motivating example}

We start by emphasizing the well-known fact that, even when the plant is linear autonomous, model-referenceadaptive-control systems are nonlinear. To that end, let us 
consider a feedback-linearizable system, in normal form, i.e.,

$$
\begin{aligned}
\dot{x}_{i} & =x_{i+1} \quad i \in[1, n-1] \\
\dot{x}_{n} & =\Phi(x)^{\top} \theta+g(x) u
\end{aligned}
$$

where $x:=\left[x_{1} \cdots x_{n}\right]^{\top}, \Phi: \mathbb{R}^{n} \rightarrow \mathbb{R}^{m}$ is a regressor function and $\theta \in \mathbb{R}^{m}$ is a vector of unknown lumped parameters. The control goal is to design $u$ such that this system behaves as the reference model

$$
\begin{aligned}
\dot{x}_{i}^{*} & =x_{i+1}^{*} \\
\dot{x}_{n}^{*} & =f\left(x^{*}\right)
\end{aligned} \quad i \in[1, n-1]
$$

or, in other words, to steer $x(t) \rightarrow x^{*}(t)$ where $x^{*}(t)$ is solution of (2). The feedback-linearizing control input that achieves this goal is $u:=g(x)^{-1}\left[f\left(x^{*}\right)-\Phi(x)^{\top} \theta-K e\right]$ with $e:=x-x^{*}$ and $K:=\left[\begin{array}{lll}\kappa_{1} & \cdots & \kappa_{n}\end{array}\right]$. In the case that the parameters $\theta$ are unknown, we use the certainty-equivalence adaptive controller

$$
\begin{aligned}
u & =g(x)^{-1}\left[f\left(x^{*}\right)-\Phi(x)^{\top} \hat{\theta}-K e\right] \\
\dot{\hat{\theta}} & =\gamma \Phi(x) \mathbf{B}^{\top} P e
\end{aligned}
$$

where $\mathrm{B}$ is part of the controllable pair $(A, \mathrm{~B}), A$ is Hurwitz by design, and $P=P^{\top}>0$ satisfies $A^{\top} P+P A=-Q$, given an arbitrary positive-definite symmetric matrix $Q$. Then, defining the parameter-estimation errors $\tilde{\theta}:=\hat{\theta}-\theta$ we see that the closed-loop system takes the form

$$
\begin{aligned}
\dot{e} & =A e+B(t, e) \tilde{\theta} \\
\dot{\tilde{\theta}} & =-\gamma B(t, e)^{\top} P e
\end{aligned}
$$

with $B(t, e):=-\mathrm{B} \Phi\left(e+x^{*}(t)\right)^{\top}$.

Clearly, the equation $\dot{e}=A e$ corresponds to that of the tracking errors under the assumption that the parameters are known, which is the starting point in certainty-equivalencebased adaptive control. If $B$ depended only on time, we would recover a well-studied system in textbooks on adaptive control, e.g., [15]. However, even in the simplest case in which (1) is linear, that is, if $\Phi(x)^{\top} \theta:=x^{\top} \theta$ and $g(x) \equiv$ const., the resulting closed-loop system is nonlinear time-varying; this is a well-documented fact [10], [2] that is some times obliterated in the literature. Thus, we see that the benefits of feedback linearization are lost in the case of uncertainties, while the use of other nonlinear control techniques, such as passivity-based, do not necessarily render the stability analysis problem significantly more complex. In either case, we are often confronted to analyze systems of the form

$$
\begin{aligned}
& \dot{x}_{1}=F\left(t, x_{1}\right)+\Phi\left(t, x_{1}\right) x_{2} \\
& \dot{x}_{2}=-\sigma \Phi\left(t, x_{1}\right)^{\top} \frac{\partial V_{1}^{\top}}{\partial x_{1}}, \quad \sigma>0,
\end{aligned}
$$

with states $x_{1} \in \mathbb{R}^{n}$ and $x_{2} \in \mathbb{R}^{m}, V_{1}: \mathbb{R}_{\geq 0} \times \mathbb{R}^{n} \rightarrow \mathbb{R}_{\geq 0}$ is a function that is (at least) one continuously differentiable and, for the purpose of existence and uniqueness of solutions, the functions $F$ and $\Phi: \mathbb{R}_{\geq 0} \times \mathbb{R}^{n} \rightarrow \mathbb{R}^{n}$, and $\frac{\partial V_{1}}{\partial x_{1}}$ are locally Lipschitz continuous in $x$, uniformly in $t$.

Systems (5), under the following standing hypotheses that we describe in detail below, are our subject of study.

\section{B. Main assumptions}

Assumption 1: There exist $\mathcal{K}_{\infty}$ functions $\beta_{1}, \beta_{2}: \mathbb{R}_{\geq 0} \rightarrow$ $\mathbb{R}_{\geq 0}$, positive definite functions $\rho_{1}, \gamma_{1}: \mathbb{R}_{\geq 0} \rightarrow \mathbb{R}_{\geq 0}$, and a positive-definite and radially-unbounded function $V_{1}: \mathbb{R}_{\geq 0} \times$ $\mathbb{R}^{n} \rightarrow \mathbb{R}_{\geq 0}$ such that

$$
\begin{gathered}
\beta_{1}\left(\left|x_{1}\right|\right) \leq V_{1}\left(t, x_{1}\right) \leq \beta_{2}\left(\left|x_{1}\right|\right) \\
\frac{\partial V_{1}}{\partial t}+\frac{\partial V_{1}}{\partial x_{1}} F\left(t, x_{1}\right) \leq-\gamma_{1}\left(\left|x_{1}\right|\right) \\
\max \left\{\left|\frac{\partial V_{1}}{\partial x_{1}}\right|,\left|F\left(t, x_{1}\right)\right|\right\} \leq \rho_{1}\left(\left|x_{1}\right|\right) .
\end{gathered}
$$

The condition (6c) simply imposes that $F(t, 0) \equiv 0$ and $F\left(t, x_{1}\right) \equiv 0$ is uniformly bounded in $t$; similarly for $\partial V_{1} / \partial x_{1}$.

More importantly, the class of systems for which Inequalities (6a) and (6b) hold, includes marginally stable systems. This follows, by a direct computation, observing that the function

$$
V_{2}(t, x):=V_{1}\left(t, x_{1}\right)+\frac{1}{2 \sigma}\left|x_{2}\right|^{2},
$$

where $x:=\left[\begin{array}{ll}x_{1}^{\top} & x_{2}^{\top}\end{array}\right]^{\top}$, is positive definite and radially unbounded and, moreover, its total derivative along the trajectories of (5) yields

$$
\dot{V}_{2}(t, x) \leq-\gamma_{1}\left(\left|x_{1}\right|\right)
$$

which is negative semi-definite. Hence, a key feature of the systems (5) under Assumption 1 is the property (8) from which, together with (6a), one can conclude uniform global stability and even infer that $x_{1} \rightarrow 0$. For instance, if $\gamma_{1}(s) \propto$ $s^{2}$ we see that $x_{1} \in \mathcal{L}_{2} \cap \mathcal{L}_{\infty}$, and $x_{2} \in \mathcal{L}_{\infty}$. In this case, standard arguments that appeal to Barbălat's lemma may be used to conclude that $x_{1} \rightarrow 0$.

In the following we construct a strict Lyapunov function for (5) by regarding this system as if composed of a nominal (simpler) part and some vanishing output-injection terms that depend on $x_{1}$. That is, let

$$
K\left(t, x_{1}\right):=\left[\Phi\left(t, x_{1}\right)-\Phi_{\circ}(t)\right] \quad \text { and } \quad \Phi_{\circ}(t):=\Phi(t, 0) .
$$

Then, the equations (5) are equivalent to

$$
\begin{aligned}
& \dot{x}_{1}=F\left(t, x_{1}\right)+\Phi_{\circ}(t) x_{2}+K\left(t, x_{1}\right) x_{2} \\
& \dot{x}_{2}=-\sigma \Phi_{\circ}(t)^{\top} \frac{\partial V_{1}^{\top}}{\partial x_{1}}-\sigma K\left(t, x_{1}\right)^{\top} \frac{\partial V_{1}^{\top}}{\partial x_{1}} .
\end{aligned}
$$

As previously discussed, in view of Assumption 1, we have $x_{1}(t) \rightarrow 0$ which, in view of (9), implies that $K\left(t, x_{1}(t)\right) \rightarrow 0$. Furthermore, since by assumption $\Phi\left(t, x_{1}\right)$ is locally Lipschitz in $x_{1}$, uniformly in $t$ and it is uniformly bounded in $t$, there exist a continuous non-decreasing function $\rho_{2}: \mathbb{R}_{\geq 0} \rightarrow \mathbb{R}_{\geq 0}$, such that

$$
\left|K\left(t, x_{1}\right)\right| \leq \rho_{2}\left(\left|x_{1}\right|\right) .
$$

On the other hand, as we shall see through an example in Section III, $\Phi_{\circ}$ is reminiscent of a function of the system's dynamics, evaluated along exogenous signals, such as reference trajectories. This is all the more significant since our second main hypothesis is inspired by the fact that persistency of excitation of $\Phi_{\circ}$, and not of $\Phi(t, x)$ as it might be supposed, is 
sufficient and necessary for uniform global asymptotic stability [18]. This brings us to our second main hypothesis.

Assumption 2: There exist real positive numbers $\mu, T$, and $\phi_{M}$ such that

$$
\begin{gathered}
\max \left\{\left|\Phi_{\circ}(t)\right|_{\infty},\left|\dot{\Phi}_{\circ}(t)\right|_{\infty}\right\} \leq \phi_{M} \\
\int_{t}^{t+T} \Phi_{\circ}(s)^{\top} \Phi_{\circ}(s) d s \geq \mu I \quad \forall t \geq 0 .
\end{gathered}
$$

\section{Main result}

Theorem 1: Consider the system (5) under Assumptions 12 . Then, the origin is uniformly globally asymptotically stable and it admits a strict Lyapunov function $\mathcal{V}: \mathbb{R}_{\geq 0} \times \mathbb{R}^{n+m} \rightarrow$ $\mathbb{R}_{\geq 0}$ defined as follows:

$$
\mathcal{V}(t, x):=2 c \sigma V_{2}(t, x)+V_{3}(t, x)+\frac{1}{4} V_{4}\left(t, x_{2}\right)+W(t, x)
$$

where $c:=\phi_{M}+\frac{\phi_{M}^{2}}{2}$,

$$
\begin{aligned}
V_{3}(t, x) & :=\sum_{i=1}^{3} \alpha_{i}\left(V_{2}(t, x)\right), \quad i \in\{1,2,3\} \\
V_{4}\left(t, x_{2}\right) & :=-\int_{t}^{\infty}\left|\Phi_{\circ}(\tau) x_{2}\right|^{2} \mathrm{e}^{t-\tau} d \tau \\
W(t, x) & :=-x_{1}^{\top} \Phi_{\circ}(t) x_{2},
\end{aligned}
$$

and $\alpha_{i}: \mathbb{R}_{\geq 0} \rightarrow \mathbb{R}_{\geq 0}$ are positive definite non-decreasing functions such that, for any $\lambda_{1}$ and $\lambda_{2}>0$, it holds that

$$
\begin{aligned}
\frac{\partial \alpha_{1}}{\partial V_{2}}\left(V_{2}(t, x)\right) \gamma_{1}\left(\left|x_{1}\right|\right) & \geq \phi_{M}\left[\rho_{2}\left(\left|x_{1}\right|\right)-\rho_{2}(0)\right]\left|x_{2}\right|^{2} \\
\frac{\partial \alpha_{2}}{\partial V_{2}}\left(\beta_{1}\left(\left|x_{1}\right|\right)\right) \gamma_{1}\left(\left|x_{1}\right|\right) & \geq \frac{\lambda_{2}}{2} \phi_{M}^{2}\left|x_{1}\right|^{2}+\frac{\lambda_{1}}{2} \rho_{1}\left(\left|x_{1}\right|\right)^{2} \\
& +\gamma_{2}^{\prime}\left(\left|x_{1}\right|\right)+\rho_{3}\left(\left|x_{1}\right|\right)\left|x_{1}\right| \\
\alpha_{3}\left(\beta_{1}\left(\left|x_{1}\right|\right)\right) & \geq c\left|x_{1}\right|^{2}
\end{aligned}
$$

where $\rho_{1}$ is defined in Assumption 1, $\rho_{2}$ is introduced in (11) and, if necessary, it is redefined to satisfy

$$
8 \rho_{2}(0) \phi_{M} \leq \mu \mathrm{e}^{-T} \text {. }
$$

In addition, the function $\rho_{3}: \mathbb{R}_{\geq 0} \rightarrow \mathbb{R}_{\geq 0}$ is defined as

$$
\rho_{3}\left(\left|x_{1}\right|\right):=\sigma \phi_{M}\left[\rho_{2}\left(\left|x_{1}\right|\right)+\phi_{M}\right] \rho_{1}\left(\left|x_{1}\right|\right)
$$

and, for any positive-definite function $\gamma_{2}: \mathbb{R}_{\geq 0} \rightarrow \mathbb{R}_{\geq 0}$,

$$
\gamma_{2}^{\prime}\left(\left|x_{1}\right|\right):=\gamma_{2}\left(\left|x_{1}\right|\right)+2 \lambda_{2} \phi_{M}^{2} \rho_{3}\left(\left|x_{1}\right|\right)^{2} .
$$

The Inequalities (15)-(17) are all met by construction while $K(t, 0) \equiv 0$ so $\rho_{2}$ may be chosen such that $\rho_{2}(0)=0$; this obviates the condition (18). Nonetheless, defining $\rho_{2}$ so that $\rho_{2}(0)>0$ be arbitrarily small may result convenient to verify Inequality (15) locally, in cases in which the growth of $\rho_{2}(s)$ is higher than that of $\gamma_{1}(s)$ near the origin - see Section III farther. Furthermore, we stress that, through $V_{2}, \alpha_{1}$ is a function of $\left|x_{2}\right|^{2}$ and since it is smooth by design, $\left|x_{2}\right|^{2}$ may be factored out of $\partial \alpha_{1} / \partial V_{2}$ using the relation

$$
\varphi(v)=v \int_{0}^{1} \frac{\partial \varphi}{\partial v}(s v) d s+\varphi(0)
$$

which holds for any smooth function $v \mapsto \varphi$. For instance, if $\alpha_{1}$ is constructed as a polynomial of degree $p$, that is,

$$
\alpha_{1}\left(V_{2}\right):=k_{1} V_{2}+k_{2} V_{2}^{2}+\cdots+k_{q} V_{2}^{p},
$$

Inequality (15) holds if there exist reals $k_{i} \geq 0$ such that

$$
\gamma_{1}\left(\left|x_{1}\right|\right) \sum_{i=2}^{p} i k_{i} \frac{i-1}{2 \sigma} \beta_{1}\left(\left|x_{1}\right|\right)^{i-2} \geq \phi_{M}\left[\rho_{2}\left(\left|x_{1}\right|\right)-\rho_{2}(0)\right]
$$

where $\beta_{1}$ is introduced in Assumption 1.

Proof of Theorem 1: First, we prove that $\mathcal{V}$ is positive definite on $\mathbb{R}^{n+m}$ and radially unbounded. Then, we show that its total derivative satisfies

$$
\dot{\mathcal{V}} \leq-\frac{1}{4}\left|\Phi_{\circ}(t) x_{2}\right|^{2}-\frac{\mu \mathrm{e}^{-T}}{8}\left|x_{2}\right|^{2}-\gamma_{2}\left(\left|x_{1}\right|\right),
$$

which is negative definite.

To establish the positivity and the radial-unboundedness of $\mathcal{V}$ we first observe that

$$
\frac{\phi_{M}}{2}\left[\left|x_{1}\right|^{2}+\left|x_{2}\right|^{2}\right] \geq W(t, x) \geq-\frac{\phi_{M}}{2}\left[\left|x_{1}\right|^{2}+\left|x_{2}\right|^{2}\right]
$$

while the function $V_{4}$ defined in (14b) satisfies

$$
-\phi_{M}^{2}\left|x_{2}\right|^{2} \leq V_{4}\left(t, x_{2}\right) \leq-\mu \mathrm{e}^{-T}\left|x_{2}\right|^{2} .
$$

Thus, after (17), we obtain

$2 \sigma c V_{2}(t, x)+\alpha_{3}\left(V_{2}(t, x)\right)+\frac{1}{4} V_{4}\left(t, x_{2}\right)+W(t, x) \geq \frac{c}{2}|x|^{2}$.

Furthermore, $\mathcal{V}(t, x)$ is decrescent because it is bounded from above by

$$
\left[\alpha_{1}+\alpha_{2}+\alpha_{3}+2 \sigma c \text { Id }\right] \circ\left(\beta_{2}\left(\left|x_{1}\right|\right)+\frac{1}{2 \sigma}\left|x_{2}\right|^{2}\right)+\phi_{M}|x|^{2}
$$

where $\operatorname{Id}(s)=s$.

We proceed now to evaluate the total derivative of $\mathcal{V}$ along the trajectories of (5). To that end, let us define $\xi:=\Phi_{\circ}(t) x_{2}$. Then, the total derivative of $W$ along the trajectories of (5) yields

$$
\begin{aligned}
\dot{W}(t, x)=-F & \left(t, x_{1}\right)^{\top} \xi-|\xi|^{2}-x_{2}^{\top} K\left(t, x_{1}\right) \Phi_{\circ}(t) x_{2} \\
& -x_{1}^{\top} \dot{\Phi}_{\circ}(t) x_{2}-\sigma x_{1}^{\top} \Phi_{\circ}(t) \Phi\left(t, x_{1}\right)^{\top} \frac{\partial V_{1}^{\top}}{\partial x_{1}}
\end{aligned}
$$

and, for any $\lambda_{1}, \lambda_{2}>0$, we have

$$
\begin{aligned}
\dot{W}(t, x) \leq & \frac{\lambda_{1}}{2} \rho_{1}\left(\left|x_{1}\right|\right)^{2}+\frac{|\xi|^{2}}{2 \lambda_{1}}-|\xi|^{2}+\phi_{M}\left|x_{2}\right|^{2} \rho_{2}\left(\left|x_{1}\right|\right) \\
& +\frac{\left|x_{2}\right|^{2}}{2 \lambda_{2}}+\frac{\lambda_{2} \phi_{M}^{2}}{2}\left|x_{1}\right|^{2} \\
& +\sigma \phi_{M}\left|x_{1}\right|\left[\rho_{2}\left(\left|x_{1}\right|\right)+\phi_{M}\right] \rho_{1}\left(\left|x_{1}\right|\right) .
\end{aligned}
$$

In the computation of the latter we used Young's inequality to bound cross-terms as well as (6c), (11), and (12a).

The total derivative of $V_{3}(t, x)$ satisfies

$$
\dot{V}_{3}(t, x) \leq-\left[\frac{\partial \alpha_{1}}{\partial V_{2}}+\frac{\partial \alpha_{2}}{\partial V_{2}}\right] \gamma_{1}\left(\left|x_{1}\right|\right) \leq 0,
$$

where we used $\left[\partial \alpha_{3} / \partial V_{2}\right] \dot{V}_{2} \leq 0$, which holds since $\alpha_{3}\left(V_{2}\right)$ is non-decreasing and $\dot{V}_{2} \leq 0$. Putting together (25), (26), and 
using (16), we see that the total derivative of $V_{3}+W$ along the trajectories of (10) yields

$$
\begin{aligned}
\dot{V}_{3}(t, x)+\dot{W}(t, x) & \leq-\left[\frac{\partial \alpha_{1}}{\partial V_{2}} \gamma_{1}\left(\left|x_{1}\right|\right)-\phi_{M} \rho_{2}\left(\left|x_{1}\right|\right)\left|x_{2}\right|^{2}\right] \\
- & {\left[1-\frac{1}{2 \lambda_{1}}\right]|\xi|^{2}-\gamma_{2}^{\prime}\left(\left|x_{1}\right|\right)+\frac{1}{2 \lambda_{2}}\left|x_{2}\right|^{2}, }
\end{aligned}
$$

so fixing $\lambda_{1}>1$, we obtain, in view of (15),

$$
\begin{aligned}
\dot{V}_{3}(t, x)+\dot{W}(t, x) \leq- & \frac{1}{2}|\xi|^{2}-\gamma_{2}^{\prime}\left(\left|x_{1}\right|\right) \\
& +\left[\frac{1}{2 \lambda_{2}}+\phi_{M} \rho_{2}(0)\right]\left|x_{2}\right|^{2} .
\end{aligned}
$$

Finally, we evaluate the total derivative of $V_{4}$ in (13) and add it to the previous expression. We have

$$
\begin{aligned}
\dot{V}_{4}\left(t, x_{2}\right)= & |\xi|^{2}+V_{4}\left(t, x_{2}\right) \\
& +2 \sigma x_{2}^{\top}\left[\int_{t}^{\infty} \Phi_{\circ}(\tau)^{\top} \Phi_{\circ}(\tau) \Phi(t, x) \mathrm{e}^{t-\tau} d \tau\right] \frac{\partial V_{1}^{\top}}{\partial x_{1}}
\end{aligned}
$$

so, using (12a), we obtain

$$
\begin{aligned}
\dot{V}_{4}\left(t, x_{2}\right) \leq-\mu \mathrm{e}^{-T}\left|x_{2}\right|^{2}+|\xi|^{2}+\frac{1}{2 \lambda_{2}}\left|x_{2}\right|^{2} & \\
& +2 \lambda_{2} \sigma^{2} \phi_{M}^{4}\left[\phi_{M}+\rho_{2}\left(\left|x_{1}\right|\right)\right]^{2} \rho_{1}\left(\left|x_{1}\right|\right)^{2} .
\end{aligned}
$$

Thus, setting $\lambda_{2}$ to satisfy

$$
\frac{1}{\lambda_{2}}<\frac{1}{8} \mu \mathrm{e}^{-T}-\phi_{M} \rho_{2}(0)
$$

and in view of (20), we obtain Inequality (23).

\section{AdAPTIVE CONTROL OF LAGRANGIAN SYSTEMS}

We illustrate the utility of Theorem 1 by presenting, as far as we know for the first time, a proof via Lyapunov's direct method of uniform global asymptotic stability for a wellknown adaptive controller for Euler-Lagrange systems. These are defined by the equations

$$
D_{\theta}(q) \ddot{q}+C_{\theta}(q, \dot{q}) \dot{q}+g_{\theta}(q)=u, \quad q \in \mathbb{R}^{n},
$$

where $D_{\theta}: \mathbb{R}^{n} \rightarrow \mathbb{R}^{n \times n}$ defines the inertia matrix which is positive definite symmetric, $C_{\theta}: \mathbb{R}^{n} \times \mathbb{R}^{n} \rightarrow \mathbb{R}^{n \times n}$ defines the Coriolis-and-centrifugal-forces matrix, which satisfies

$$
\dot{D}_{\theta}(q)=C_{\theta}(q, \dot{q})+C_{\theta}(q, \dot{q})^{\top} .
$$

The function $g_{\theta}: \mathbb{R}^{n} \rightarrow \mathbb{R}^{n}$ defines the gravity forces vector. All these functions depend, in addition to the generalized positions $q$ and generalized velocities $\dot{q}$, on lumped constant parameters $\theta \in \mathbb{R}^{m}$. It is assumed, moreover, that $D_{\theta}$, $C_{\theta}$ and $g_{\theta}$ are linear in $\theta$ that is, there exists a function $\Upsilon: \mathbb{R}^{3 n} \rightarrow \mathbb{R}^{m \times n}$ such that the right hand side of (27) equals to $\Upsilon(q, \dot{q}, \dot{q})^{\top} \theta$. Furthermore, in view of (28), $C_{\theta}$ is linear in $\dot{q}$ and, as it is customary, it is assumed that $D_{\theta}$ and $C_{\theta}$ is uniformly bounded for all $q \in \mathbb{R}^{n}$. More precisely, there exist $d_{m}, d_{M}$, and $k_{c}>0$, such that, for all $q, \dot{q} \in \mathbb{R}^{n}$, and $\theta \in \mathbb{R}^{m}$,

$$
\begin{gathered}
d_{m} \leq\left|D_{\theta}(q)\right| \leq d_{M}, \\
\left|C_{\theta}(q, \dot{q})\right| \leq k_{c}|\dot{q}| .
\end{gathered}
$$

The adaptive state-feedback tracking control problem for (27) consists in the following. Given a sufficiently smooth reference trajectory $t \mapsto q_{d}$, bounded and with bounded derivatives, find an adaptive controller so that $\tilde{q}:=q-q_{d}$ and $\dot{\tilde{q}}:=\dot{q}-\dot{q}_{d}$ tend to zero asymptotically. This problem is solved, at least, since the seminal paper [24] where the (non-uniform) convergence of the tracking errors is established and, under a condition of persistency of excitation, in [29], it is showed that the parameter estimation errors also converge to zero. In [30] a strict Lyapunov function, with which global asymptotic stability is established, is given for the non-adaptive case; see also [31] for a statement on global exponential stability. To the best of our knowledge, the first proof of uniform global asymptotic stability in the adaptive case (hence, including uniform convergence of the parameter estimation errors) under the action of the controller proposed in [24] was established in [32], under a condition on persistency of excitation along the reference trajectories. Here, we present, as far as we know for the first time, a proof of uniform global asymptotic stability for the adaptive controller of [24], via Lyapunov's direct method.

We recall that the adaptive controller of [24] is given by

$$
\begin{aligned}
u & =D_{\hat{\theta}} \ddot{q}_{r}+C_{\hat{\theta}}(q, \dot{q}) q_{r}+g_{\hat{\theta}}(q)-k_{d} s \\
\dot{\hat{\theta}} & =-\sigma \Psi(t, \tilde{q}, s) s, \quad \sigma>0
\end{aligned}
$$

where $s:=\dot{q}_{r}-\dot{q}, \dot{q}_{r}:=\dot{q}_{d}(t)-\lambda \tilde{q}$, and $\lambda, k_{d}$ are positive constants. Furthermore, $\hat{\theta}$ is an estimate of the unknown lumped parameters $\theta$ and $\Psi$ is the so-called regressor function which is defined by the identity

$$
D_{\tilde{\theta}}(q) \ddot{q}_{r}+C_{\tilde{\theta}}(q, \dot{q}) \dot{q}_{r}+g_{\tilde{\theta}}(q)=: \Psi(t, \tilde{q}, s)^{\top} \tilde{\theta}
$$

which holds in view of the linearity of $D_{\theta}, C_{\theta}$ and $g_{\theta}$ with respect to $\theta$. For the purpose of analysis, we stress that the regressor $\Psi$ is a function of $(t, \tilde{q}, s)$; indeed, we observe that even though the terms on left-hand side of (31) are written as functions of $q, \dot{q}, \ddot{q}_{r}$, and $\dot{q}_{r}$, the latter are, in turn, functions of the closed-loop variables $\tilde{q}$ and $s$, as well as time, through the reference trajectories $\dot{q}_{d}(t)$ and its derivatives. That is,

$$
\begin{aligned}
q & =\tilde{q}+\dot{q}_{d}(t), & \dot{q} & =\dot{q}_{d}(t)-\lambda \tilde{q}-s, \\
\dot{q}_{r} & =\dot{q}_{d}(t)-\lambda \tilde{q}, & \ddot{q}_{r} & =\ddot{q}_{d}(t)-\lambda s+\lambda^{2} \tilde{q} .
\end{aligned}
$$

Thus, with an abuse of notation, we write the closed-loop dynamics of (27) with (30) as

$$
\begin{aligned}
D_{\theta}(q) \dot{s}+C_{\theta}(q, \dot{q}) s+k_{d} s & =\Psi(t, \tilde{q}, s)^{\top} \tilde{\theta} \\
\dot{\tilde{q}} & =-\lambda \tilde{q}+s \\
\dot{\tilde{\theta}} & =-\sigma \Psi(t, \tilde{q}, s) s .
\end{aligned}
$$

Now, as it was established in [30], a direct computation shows that the total derivative of the function

$$
V(t, \tilde{q}, s):=\frac{1}{2} s^{\top} D_{\theta}(q) s+\frac{\lambda k_{d}}{2}|\tilde{q}|^{2}+\frac{1}{2 \sigma}|\tilde{\theta}|^{2}
$$

along the trajectories of (32) yields

$$
\dot{V}(t, \tilde{q}, s) \leq-\frac{1}{2}\left[k_{d}|s|^{2}+\lambda k_{d}|\tilde{q}|^{2}\right] .
$$

Remark 1: Once more, in the previous expressions we used the familiar notation $D_{\theta}(q)$ instead of the more appropriate, but cumbersome one, $D_{\theta}\left(\tilde{q}+q_{d}(t)\right)$.

This is the starting point to establish a direct proof of the following statement, using Lyapunov's first method $-c f$. [32]. 
Proposition 1: Consider the system (27) and assume that the inequalities in (29) hold. For the reference trajectories $t \mapsto$ $q_{d}$ assume that there exists $\beta_{d}>0$ such that

$$
\max \left\{\left|\ddot{q}_{d}\right|_{\infty},\left|\dot{q}_{d}\right|_{\infty},\left|q_{d}\right|_{\infty}\right\} \leq \beta_{d}
$$

and there exist $T$ and $\mu>0$ such that

$$
\int_{t}^{t+T} \Psi(\tau, 0,0) \Psi(\tau, 0,0)^{\top} d \tau \geq \mu I \quad \forall t \geq 0 .
$$

Then, the origin in the state space of the closed-loop system (32), that is $\{(\tilde{q}, s, \tilde{\theta})=(0,0,0)\}$, is uniformly globally asymptotically stable.

Remark 2: We stress that $\Psi(t, 0,0)$ is a matrix that depends only on the reference trajectories and its derivatives and solves the identity

$$
D_{\tilde{\theta}}\left(q_{d}\right) \ddot{q}_{d}+C_{\tilde{\theta}}\left(q_{d}, \dot{q}_{d}\right) \dot{q}_{d}+g_{\tilde{\theta}}\left(q_{d}\right)=: \Psi(t, 0,0)^{\top} \tilde{\theta}
$$

in which we dropped the argument $(t)$ from $q_{d}(t)$ and its derivatives. Moreover, the condition (35) is also necessary for uniform global asymptotic stability [32].

The statement of Proposition 1 is a consequence of the following original result.

Proposition 2: The closed-loop system (32), under the conditions of Proposition 1, admits the strict Lyapunov function

$$
\begin{aligned}
\mathcal{V}(t, \tilde{q}, s, \tilde{\theta}):= & \sum_{j=1}^{3} \delta_{j}\left[\frac{1}{2} s^{\top} D_{\theta}(q(t)) s+\frac{\lambda k_{d}}{2}|\tilde{q}|^{2}+\frac{1}{2 \sigma}|\tilde{\theta}|^{2}\right]^{j} \\
& -\int_{t}^{\infty}\left|D_{\theta}\left(q_{d}(\tau)\right)^{-1} \Psi(\tau, 0,0)^{\top} \tilde{\theta}\right|^{2} \mathrm{e}^{t-\tau} d \tau \\
& -s^{\top} D_{\theta}\left(q_{d}(t)\right)^{-1} \Psi(t, 0,0)^{\top} \tilde{\theta}
\end{aligned}
$$

which is positive-definite, radially-unbounded, and its derivative satisfies

$$
\dot{\mathcal{V}}(t, \tilde{q}, s, \tilde{\theta}) \leq-\frac{1}{2} \gamma_{1}^{\prime}\left[|s|^{2}+|\tilde{q}|^{2}\right]-\frac{1}{8} \mu \mathrm{e}^{-T}|\tilde{\theta}|^{2},
$$

where $\gamma_{1}^{\prime}:=\left(k_{d} / 2\right) \min \{1, \lambda\}$, for sufficiently large values of the constant parameters $\delta_{j}>0$.

Proof: The construction of $\mathcal{V}$ follows by verifying the conditions of Theorem 1. The first step is to write the closedloop equations in the form (5). To that end, let

$$
x_{1}:=\left[\begin{array}{ll}
\tilde{q} & s
\end{array}\right]^{\top}, \quad x_{2}:=\tilde{\theta} .
$$

Then, since $D_{\theta}(q)$ is invertible for all $q \in \mathbb{R}^{n}$ we may define

$$
\begin{aligned}
& F\left(t, x_{1}\right):=\left[\begin{array}{cc}
-\lambda I & I \\
0 & {\left[D_{\theta}^{-1} C_{\theta}+k_{d} I\right]}
\end{array}\right]\left[\begin{array}{l}
\tilde{q} \\
s
\end{array}\right] \\
& \Phi\left(t, x_{1}\right)=\left[\begin{array}{c}
0 \\
D_{\theta}^{-1} \Psi(t, \tilde{q}, s)^{\top}
\end{array}\right]
\end{aligned}
$$

in which, to simplify the notation, we dropped the arguments of $D_{\theta}\left(\tilde{q}+q_{d}(t)\right)^{-1}$ and $C_{\theta}\left(\tilde{q}+q_{d}(t), \dot{q}_{d}(t)-\lambda \tilde{q}-s\right)$.

The function $V_{1}$ in Assumption 1 corresponds to the first two terms of the function $V$ in (33) and corresponds to the function proposed in [30], which we repeat here for convenience:

$$
V_{1}\left(t, x_{1}\right):=\frac{1}{2} s^{\top} D_{\theta}(q(t)) s+\frac{\lambda k_{d}}{2}|\tilde{q}|^{2} .
$$

That is,

$$
\frac{\partial V_{1}^{\top}}{\partial x_{1}}=\left[\begin{array}{c}
\lambda k_{d} \tilde{q} \\
D_{\theta}(q(t)) s
\end{array}\right],
$$

so (32c) has exactly the form (5b) and, actually, the system (32) has the form of (5).

Now we verify Assumption 1. On one hand, the inequalities in (6a) follow from (29) with

$$
\beta_{1}\left(\left|x_{1}\right|\right):=\beta_{1}^{\prime}\left|x_{1}\right|^{2}, \quad \beta_{2}\left(\left|x_{1}\right|\right):=\beta_{2}^{\prime}\left|x_{1}\right|^{2}
$$

where

$$
\beta_{1}^{\prime}:=\frac{1}{2} \min \left\{d_{m}, \lambda k_{d}\right\} \quad \beta_{2}^{\prime}:=\frac{1}{2} \max \left\{d_{M}, \lambda k_{d}\right\} .
$$

On the other hand, a direct computation using (28) shows that (6b) holds with $\gamma_{1}\left(\left|x_{1}\right|\right):=\gamma_{1}^{\prime}\left|x_{1}\right|^{2}$ and, after (29a),

$$
\left|\frac{\partial V_{1}}{\partial x_{1}}\right| \leq \rho_{1}^{\prime}\left|x_{1}\right|, \quad \rho_{1}^{\prime}:=\max \left\{d_{M}, \lambda k_{d}\right\} .
$$

In addition, a simple inspection of (39) and the inequalities in (29) shows that $F\left(t, x_{1}\right)$ is of order $\left|x_{1}\right|^{2}$ for large $\left|x_{1}\right|$ and of order $\left|x_{1}\right|$ locally. Hence, (6c) holds with

$$
\rho_{1}\left(\left|x_{1}\right|\right):=\rho_{11}\left|x_{1}\right|+\rho_{12}\left|x_{1}\right|^{2},
$$

where $\rho_{11} \geq \rho_{1}^{\prime}$ and $\rho_{12}$ depend on $\beta_{d}$ in (34) as well as on $d_{m}$ and $k_{c}$ in (29).

Next, we verify Assumption 2. Inequality (12a) holds because $\Phi_{\circ}$ and $\dot{\Phi}_{\circ}$ are continuous functionals of $q_{d}, \dot{q}_{d}$, and $\ddot{q}_{d}$, which are bounded - see (34), while (12b) is equivalent to (35) since $D_{\theta}(\cdot)^{-1}$ is full rank.

Now we proceed to construct three functions $\alpha_{1}, \alpha_{2}$, and $\alpha_{3}$, satisfying (15)-(17). The fact that $\beta_{1}, \beta_{2}, \rho_{1}$, and $\gamma_{1}$ are polynomials motivates a similar choice for the functions $\alpha_{i}$. To determine their respective orders (so that (15)-(17) hold) it is left to find an appropriate function $\rho_{2}$ which, in turn, shall determine $\rho_{3}$. For this, we see that all the functions on the left-hand side of (27) are at most of quadratic growth hence, in view of (29) and (34), there exist $\rho_{21}$ and $\rho_{22}>0$ such that

$$
|\Psi(t, \tilde{q}, s)-\Psi(t, 0,0)| \leq \rho_{21}\left|x_{1}\right|+\rho_{22}\left|x_{1}\right|^{2} .
$$

This implies that (11) holds with $\rho_{2}\left(\left|x_{1}\right|\right)=\rho_{21}\left|x_{1}\right|+$ $\rho_{22}\left|x_{1}\right|^{2}$. Nonetheless, another simple inspection shows that with this choice of $\rho_{2}$, the condition (15) may not hold locally because the left-hand side of this inequality is at least of 2nd order in $\left|x_{1}\right|$ (due to the factor $\gamma_{1}$ ) while the right-hand side contains a first-order term of $\left|x_{1}\right|$. Therefore, we use the inequality $\left|x_{1}\right| \leq 1+\left|x_{1}\right|^{2}$ to redefine $\rho_{2}$ as

$$
\rho_{2}\left(\left|x_{1}\right|\right):=\frac{\varepsilon \rho_{21}}{2}+\left[\frac{\rho_{21}}{2 \varepsilon}+\rho_{22}\right]\left|x_{1}\right|^{2}, \quad \varepsilon>0 .
$$

With this new choice of $\rho_{2}$ (11) still holds and, moreover, Inequality (22) holds with $p=2$, for sufficiently large $k_{2}$; in turn, this implies (15). It is to be noted that the choice of $\varepsilon>0$ is determined by (18); this lifts any possible restriction on the parameters $\mu$ and $T$, which are given. Thus, we conclude that defining $\alpha_{1}\left(V_{2}\right)$ as a second-order polynomial of $V_{2}$ will do.

Next, we construct $\alpha_{2}$ so that Inequality (16) hold. Let $\gamma_{2}\left(\left|x_{1}\right|\right):=(1 / 2) \gamma_{1}^{\prime}\left|x_{1}\right|^{2}$. A simple inspection, using (43) and 
(45), shows that $\rho_{3}$ in (19) is a polynomial of 4 th order, hence the right-hand side of (16) is bounded from above by

$$
\sum_{j=2}^{5} \varrho_{j}\left|x_{1}\right|^{j}, \quad \varrho_{j}>0 .
$$

Therefore, using the triangle inequality

$$
\varrho_{j}\left|x_{1}\right|^{j} \leq \frac{1}{2}\left[\varrho_{j}\left|x_{1}\right|^{j-1}+\varrho_{j}\left|x_{1}\right|^{j+1}\right]
$$

for $j=3$ and $j=5$, we see that (16) holds if $\alpha_{2}\left(V_{2}\right)$ is a polynomial of 3 rd order that is, if

$$
\alpha_{2}\left(V_{2}\right):=\sum_{j=1}^{3} k_{j} V_{2}^{j} .
$$

Indeed, if such is the case,

$$
\frac{\partial \alpha_{2}}{\partial V_{2}}\left(\beta_{1}^{\prime}\left|x_{1}\right|^{2}\right) \gamma_{1}^{\prime}\left|x_{1}\right|^{2} \geq \gamma_{1}^{\prime} \sum_{j=1}^{3} j k_{j} \beta_{1}^{\prime j-1}\left|x_{1}\right|^{2 j}
$$

so (16) holds provided that $k_{j}$ are sufficiently large.

The term $\alpha_{3}\left(V_{2}\right)$ is designed to make $\mathcal{V}$ positive definite and radially unbounded. Inequality (17) trivially holds with $\alpha_{3}\left(\beta_{1}\right):=\left(c / \beta_{1}^{\prime}\right) \beta_{1}$. Finally, from (40) we see that $V_{4}$ and $W$ correspond to the last two terms on the right-hand side of (37) respectively.

Thus, all the conditions of Theorem 1 hold with the function $\mathcal{V}$ in (37), which is of the form (13), $\alpha_{1}, \alpha_{2}$, and $\alpha_{3}$ being polynomials in $V_{2}$ of second, third, and first order respectively, and the inequality (23) corresponds in this case to (38).

\section{CONCLUSIONS}

We have provided a relatively simple and intuitive strict Lyapunov function for nonlinear time-varying systems that appear in the context of passivity-based adaptive control. We believe that the construction provided here may serve as a building block in the solution of other complex problems such as adaptive observer-based tracking control. In that regard, we provided a strict Lyapunov function for a well-known controller that is recurrently used in its original or in modified forms, in a variety of more modern control problems such as formation control and synchronization.

\section{REFERENCES}

[1] K. Narendra and A. Annaswamy, "Robust adaptive control in the presence of bounded disturbances," IEEE Trans. on Automat. Contr., vol. 31, no. 4, pp. 306-315, 1986.

[2] H. Khalil, Nonlinear systems. New York: Macmillan Publishing Co., 2nd ed., 1996.

[3] Barbălat, "Systèmes d'équations différentielles d'oscillations non linéaires," Revue de Mathématiques Pures et Appliquées, vol. Vol. 4, no. 2, pp. 267-270, 1959. Académie de la République Populaire Roumaine (in French).

[4] K. J. Åström and Bohn, "Numerical identification of linear dynamic systems from normal operating records," in Proc. of the 2nd IFAC Symp. on Theory of Self-adaptive Control Systems (P. H. Hammond, ed.), (Nat. Phys. Lab., Teddington, England), pp. 96-111, 1965.

[5] B. D. O. Anderson, "Exponential stability of linear equations arising in adaptive identification," IEEE Trans. on Automat. Contr., vol. 22, no. 1, pp. 83-88, 1977.

[6] A. P. Morgan and K. S. Narendra, "On the stability of nonautonomous differential equations $\dot{x}=[A+B(t)] x$ with skew-symmetric matrix $B(t)$," SIAM J. on Contr. and Opt., vol. 15, no. 1, pp. 163-176, 1977.
[7] A. P. Morgan and K. S. Narendra, "On the uniform asymptotic stability of certain linear nonautonomous differential equations," SIAM J. on Contr. and Opt., vol. 15, no. 1, pp. 5-24, 1977.

[8] T. C. Lee and B. S. Chen, "A general stability criterion for timevarying systems using a modified detectability condition," IEEE Trans. on Automat. Contr., vol. 47, no. 5, pp. 797-802, 2002.

[9] A. Loría E. Panteley, D. Popovic, and A. Teel, "A nested Matrosov theorem and persistency of excitation for uniform convergence in stable non-autonomous systems," IEEE Trans. on Automat. Contr., vol. 50, no. 2, pp. 183-198, 2005.

[10] K. S. Narendra and A. M. Annaswamy, Stable adaptive systems. New Jersey: Prentice-Hall, Inc., 1989.

[11] A. Loría and E. Panteley, "Uniform exponential stability of linear timevarying systems:revisited," Syst. \& Contr. Letters, vol. 47, no. 1, pp. 1324, 2002.

[12] B. D. O. Anderson, R. Bitmead, C. Johnson, Jr., P. Kokotović, R. Kosut, I. Mareels, L. Praly, and B. Riedle, Stability of adaptive systems. Cambridge, MA, USA: The MIT Press, 1986.

[13] T. C. Lee, "On the equivalence relations of detectability and PE conditions with applications to stability analysis of time-varying systemss," in Proc. IEEE American Control Conference, June 2003.

[14] K. Narendra and A. Annaswamy, "Persistent excitation in adaptive systems," Int. J. of Contr., vol. 45, no. 1, pp. 127-160, 1987.

[15] P. Ioannou and J. Sun, Robust adaptive control. New Jersey, USA: Prentice Hall, 1996.

[16] A. Loría, "Explicit convergence rates for MRAC-type systems," Automatica, vol. 40, no. 8, pp. 1465-1468, 2004

[17] T. E. Gibson and A. M. Annaswamy, "Adaptive control and the definition of exponential stability," in Proc. IEEE American Control Conference, pp. 1549-1554, 2015

[18] A. Loría E. Panteley, D. Popovic, and A. Teel, " $\delta$-persistency of excitation: a necessary and sufficient condition for uniform attractivity," in Proc. 41th. IEEE Conference on Decision and Control, (Las Vegas, CA, USA), pp. 3506-3511, 2002. Paper no. REG0623.

[19] F. Mazenc, M. de Queiroz, and M. Malisoff, "Uniform global asymptotic stability of a class of adaptively controlled nonlinear systems," IEEE Trans. on Automat. Contr., vol. 54, no. 5, pp. 1152-1158, 2009.

[20] F. Mazenc, "Strict Lyapunov functions for time-varying systems," Automatica, vol. 39, pp. 349-353, 2003.

[21] M. Malisoff and F. Mazenc, Constructions of Strict Lyapunov functions. London: Springer Verlag, 2009.

[22] F. Mazenc, M. Malisoff, and O. Benard, "A simplified design for strict Lyapunov functions under Matrosov conditions," IEEE Trans. on Automat. Contr, vol. 54, no. 1, pp. 177-183, 2009.

[23] F. Mazenc and D. Nesić, "Lyapunov functions for time varying systems satisfying generalized conditions of Matrosov theorem," in Proc. of the 44th IEEE Conference on Decision and Control, pp. 5432-5437, 2005.

[24] J. J. Slotine and W. Li, "Adaptive manipulator control: a case study," IEEE Trans. on Automat. Contr., vol. AC-33, pp. 995-1003, 1988.

[25] E. Nuño, R. Ortega, L. Basañez, and D. Hill, "Synchronization of networks of nonidentical euler-lagrange systems with uncertain parameters and communication delays," IEEE Trans. on Automat. Contr., vol. 56 , pp. 935-941, April 2011.

[26] S. J. Chung and J. J. E. Slotine, "Cooperative robot control and concurrent synchronization of lagrangian systems," IEEE Transactions on Robotics, vol. 25, pp. 686-700, June 2009.

[27] A. Abdessameud, A. Tayebi, and I. G. Polushin, "Leader-follower synchronization of euler-lagrange systems with time-varying leader trajectory and constrained discrete-time communication," IEEE Trans. on Automat. Contr., vol. 62, pp. 2539-2545, May 2017.

[28] M. Lu and L. Liu, "Leader-following consensus of multiple uncertain euler-lagrange systems subject to communication delays and switching networks," IEEE Trans. on Automat. Contr., pp. 1-1, 2017.

[29] J. Slotine and W. Li, "Theoretical issues in adaptive manipulator control," in 5th Yale Workshop on Apl. Adaptive Systems Theory, pp. 252 258, 1987.

[30] M. W. Spong, R. Ortega, and R. Kelly, "Comments on "Adaptive Manipulator Control: A Case Study"," IEEE Trans. on Automat. Contr., vol. 35, no. 6, pp. 761-762, 1990.

[31] E. Yaz, "Comments on "on the robust control of robot manipulators by m.w. spong," IEEE Trans. on Automat. Contr., vol. 38, no. 3, pp. 511512, 1993.

[32] A. Loría R. Kelly, and A. Teel, "Uniform parametric convergence in the adaptive control of mechanical systems," European J. of Contr., vol. 11, no. 2 , pp. 87-100, 2005. 